\title{
On observations of correlation functions and power spectra in rain: obfuscation by advection and sampling
}

\author{
A. R. Jameson ${ }^{1,2}$ (D) \\ Received: 26 November 2019 / Accepted: 29 July 2020 / Published online: 8 August 2020 \\ (c) The Author(s) 2020
}

\begin{abstract}
Rain is often characterized using statistical approaches. Among the most common are temporal correlations and power (variance) spectra from time series measurements at a single location. Likewise, temporal observations over a network are used to deduce a radial distribution function and spatial power spectra. In such studies the potential effects of advection on the results are ignored. Moreover, observations involve filtering of the data. In time, this may involve sampling over a sufficiently long period so as to increase statistical confidence in the measurement. The same is also true for spatial observations over a network which must contain a sufficient number of instruments for a reliable characterization of the spatial variability. This also usually includes some form of averaging over time as well. Temporal averaging amounts to a low pass filter that attenuates contributions from higher frequencies. In contrast, the finite dimension of a network acts as a high-pass filter that tends to suppress the lower wavenumbers much larger than the dimension of the network. In this work the effects of both the advection of the rain and the observational filtering are considered for the simplest case of wide-sense statistically stationary and homogeneous rain along one-dimension for rain exponentially correlated in both space and time. It is found that advection and filtering can significantly shift the portrayal of the rain from the true structures. Consequently, rainfall characterizations from observations should not be over-generalized to other situations.
\end{abstract}

\section{Introduction}

Rain is obviously very important to humankind as both a sustainer of agriculture and also as a destroyer through flooding. Consequently, there has always been a lot of interest in characterizing rain not only to maximize its agricultural benefits through soil erosion management, irrigation and water storage but also to minimize its occasionally destructive impacts through flood abatement planning and forecasting on many different scales. This is not an easy task because rain is a multi-dimensional random variable in time and space. In order to reduce some of this variability, historically, there has been an on-going search for commonalities among various rain events. Because of its randomness, this has, naturally, involved investigations into the statistical properties of rain to include correlations in space and in time

Responsible Editor: Clemens Simmer.

A. R. Jameson

RJH Scientific, Inc., El Cajon, CA, USA

Arlington, USA
(Zawadzki 1973; Errico 1985; Berndtsson and Niemczynowicz 1988; Crane 1990; Germann and Zawadzki 2002; de Lima et al. 2012; Wong and Skamarock 2016) as well as calculations of power (variance) spectra for different types of rain. This latter variable is of particular value when attempting to scale rain observations and outputs from numerical models to different dimensions (Ahrens and Beck 2008; Ochoa-Rodriguez et al. 2015). Hence, for such applications there have been many studies characterizing the spatial and temporal correlations of rain as well as their associated power spectra (e.g., Zawadzki 1973; Errico 1985; Berndtsson and Niemczynowicz 1988; Bell et al. 1990; Crane 1990; Harris et al. 2001; Germann and Zawadzki 2002; Parodi et al. 2011; de Lima et al. 2012; Ochoa-Rodriguez et al. 2015; Wong and Skamarock 2016).

Typically, correlations and power spectra are computed using an ensemble of observations at a single location or over a network of instruments. At a single location, observations are usually in the form of a time series which is then processed to derive, say, the temporal correlation function and power spectra. Over networks, observations are usually averaged in time in order to help reduce fluctuations, but then they are combined to yield estimates of the radial 
correlation function (e.g. see Kostinski and Jameson 2000) and, in some cases, power spectra as well, particularly for rainfall scaling studies (Simpson and Woodley 1975; Krajewski and Duffy 1988; Bell et al. 1990; Crane 1990; Short et al. 1993; Rosenfeld et al. 1994; Sauvageot 1994; Seed et al. 1999; Krajewski et al. 2003; Steiner and Smith 2004; Rebora et al. 2006a, b; Ciach and Krajewski 2006; Larsen et al. 2010; Tokay and Bashor 2010; Jaffrain and Berne 2012; Jameson and Larsen 2016; Raupach and Berne 2016).

In reality, such studies have not only ignored the role of filtering of the observations, that is observation intervals as well as the finite dimension of networks act to filter the spectral components of a time series or spatial series of observations as described in detail in (Jameson 2017). These studies have also ignored the complexity introduced by statistical heterogeneity and by advection of the precipitation. These are important features of rain as revealed in the analyses of radar data by Zawadzki (1973) and in the numerical simulations of Leblois and Creutin (2013), for example. In such conditions the interpretations of power spectra are suspect. Specifically, when statistical heterogeneity is occurring, the power spectra lose all generality (Jameson 2019). The question becomes, "When power spectra should be generally applicable because of statistical homogeneity, can advection and filtering undermine this generality?"

Throughout this work it is assumed that over small enough domains, the temporal and spatial changes in the rain are the consequence of the evolution of the rain in the vertical as well as its horizontal transport by advection leading to precipitation streaks such as those evident in Fig. 1a, b. In this case, then, spatial refers to a one-dimensional line to at the surface while temporal refers to a one-dimensional line in time. Figure 1a shows a thunderstorm with several distinct rain streaks (it is known that this is rain because the original photograph contained a rainbow that has been removed from Fig. 1a). Obviously, precipitation is a complicated process involving different sedimentation velocities for different drop sizes spatially altered as well by vertical shear of the horizontal wind. In Fig. 1a, the vertical dimension is taken to represent what is happening in time so that the changes in optical brightness can be considered to be representative of temporal variations of rain intensity in many of the individual rain shafts. This can also be observed as well in many of the time-lapse rain shaft videos that can be found on the internet. These temporal fluctuations are then being independently advected horizontally by the wind. The spatial distribution of the rain streaks themselves is obviously representative of the spatial structure so that the rainfall rate at the surface represent both of these temporal and spatial structures.

Figure $1 b$ is a plot of a steady-state pattern of rainfall rate produced by a fixed source (generating cell) of constant precipitation at the top of the plot dropping particles at a steady

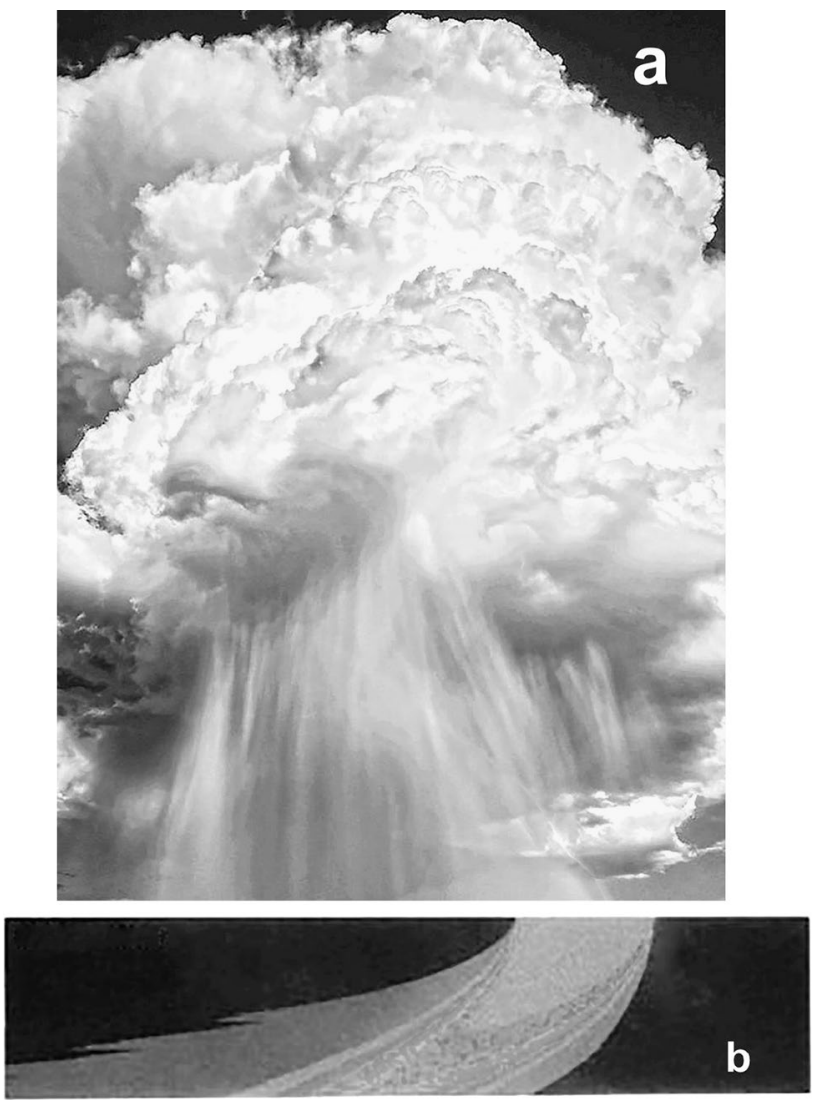

Fig. 1 a A Colorado rain shaft observed while on College of DuPage's Storm Chasing Trip 3. Photographer: Jared Rackley. Taken on May 27, 2014. Credit: NOAA Weather in Focus Photo Contest 2015. https://creativecommons.org/licenses/by/2.0/legalcode. This picture has been cropped, converted to black and white and enhanced to emphasize the rain shafts. Note the vertical temporal structure (time) as well as the obvious horizontal structure. b A numerical simulation of the rainfall pattern produced by a generating cell with precipitation falling into a wind sheared environment as discussed in the text

rate into a wind-sheared environment (from Fig. 3.3 and described in further detail in Jameson and Johnson 1990) so that the generating cell is moving the fastest. Consequently, the rain is stretched out in space to degrees depending upon the observation altitude as well as the advection speed of the generating cell. The patterns near the surface can then be identified as the statistically homogeneous spatial rain structure. Now suppose the generating cell rain was also changing, say, sinusoidally in time. The spatial pattern and mean value would then remain the same, but the temporal changes would appear as independent sinusoidal oscillations in the contour levels. Thus, in this work we consider time and space to be statistically independent. Hence, a time series of the rainfall rate at a location will reflect the advection of the intrinsic spatial structure (Fig. 1b) as well as the advection of temporal changes that are occurring in the generating cell, for example. The same will also be true 
for an observation network that, in many respects, can be considered to be just a large point measurement instrument.

In this study, then, we assume the rain to be statistically homogeneous and stationary.

This is useful because the effect of simple one-dimensional advection can be addressed analytically. It is shown below that even in such simple conditions, advection and filtering obscure the true correlation functions and power spectra often sought for practical applications. That is, the results that suggest that while the measurements of correlations and power spectra are easy, their interpretations as intrinsic, true characterizations of the rain are difficult even in the absence of statistical heterogeneity and non-stationarity.

\section{Theory}

As just discussed, in this study we only consider wide-sense statistically stationary and statistically spatially homogeneous rain (both indicated by WSS). That is, the mean values and variance spectra do not depend upon where and when they are measured. To reiterate, consider a single instrument or network when there is no advection. The instruments, then, will only see the temporal variance at its location corresponding to the length of the observations as filtered by the time window. They will not see any of the spatial fluctuations. Now consider when there is advection. A single instrument (rain gage or network) will now see temporal fluctuations induced by the passage of spatial structures (variance) over the instrument (see Fig. 1) so that fluctuations in its time series now consists of two components, namely the intrinsic temporal fluctuations and those caused by the advection of the spatial fluctuations over the instrument.

As argued in the previous section, the spatial and temporal structures are considered to be statistically independent in this study. A physical reason for this independence is that the temporal structure is predominately the consequence of raindrop evolution so that the rainfall temporal changes are in the vertical. On the other hand, in the orthogonal horizontal dimension, the spatial structure of the rain is largely governed by two-dimension dynamics of, say, the convection, as well illustrated in Fig. 1. Consequently, it is reasonable to treat the rainfall structures in time and space as being statistically independent variables so that their variances are additive.

It then follows from basic Fourier transform theory that if the total variance of a variable $x$ is $\sigma_{x}^{2}(t)$, it is also the integral over the power spectrum $S$ so that $\sigma_{x}^{2}(t)=\int S_{x}(\omega) \mathrm{d} \omega$, for example, while for another statistically independent random variable y is $\sigma_{y}^{2}(t)=\int S_{y}(\omega) \mathrm{d} \omega$. Then, since $x$ and $y$ are statistically independent, it that $\sigma_{x}^{2}(t)+\sigma_{y}^{2}(t)=\int S_{x}(\omega) \mathrm{d} \omega+\int S_{y}(\omega) \mathrm{d} \omega=\int\left[S_{x}(\omega)+S_{y}(\omega)\right] \mathrm{d} \omega$ so that the power spectra are also additive. This is true for spatial power spectra as well, of course. Moreover, if the data are wide-sense stationary and/or statistically homogeneous, the Wiener-Khintchine theorem (Wiener 1930; Khintchine 1934) shows that the power spectra and the autocorrelation functions are Fourier transform pairs so that the respective autocorrelation functions of $x$ and $y$ also add.

Before beginning the more detailed theoretical discussion, for the convenience of the reader, a list of mathematical symbols used in this section is provided in Table 1. Spatial power spectra are expressed in terms distance (wave number $\kappa$ or its inverse wavelength $\lambda$ ) while time spectra are written in terms of temporal frequency $\omega$. For spectra to be summed, they must have the same units so that for spatial spectra contributing to temporal spectra so that, $\kappa \rightarrow \omega / v$ while for temporal spectra contributing to the spatial spectra, $\omega \rightarrow \kappa \times v$.

The purpose of this study was to consider such effects for one model of precipitation in which the independent temporal and spatial correlation functions are decreasing exponential functions. Other models are possible, of course, such as the modified exponential functions in Ciach and Krajewski (2006) and Jaffrain and Berne (2012), but since all correlation functions for rain decrease with increasing lags, it can be argued that many of the effects derived using this model have general applicability even though some of the specifics will be model dependent. Moreover, we only consider localized advection so that it can be well represented by a constant velocity. Furthermore, for simplicity we will also only consider a one-dimensional line of detectors so that the advection velocity $v$ is then just the velocity component along that line.

With these considerations, the temporal and spatial correlation functions are, respectively,

$\rho(\tau)=\exp \left(-\frac{\tau}{\mathfrak{T}}\right)$

and

$\rho(s)=\exp \left(-\frac{s}{\mathfrak{Q}}\right)$,

where $\tau$ is the temporal lag while $\mathfrak{I}$ and $\mathfrak{R}$ are the respective $1 / e$ decorrelation lengths. Each of these correlation functions are then associated with their power (variance) spectra through the Wiener-Khintchine theorem vis a vis the Fourier transform (Wiener 1930; Khintchine 1934) applicable to wide-sense statistically stationary and homogeneous data. Hence,

$S(\omega)=\frac{2 \mathfrak{T}^{2}}{\pi\left(1+\mathfrak{I}^{2} \omega^{2}\right)^{2}}$

$S(\kappa)=\frac{2 \mathfrak{Q}^{2}}{\pi\left(1+\mathfrak{Q}^{2} \kappa^{2}\right)^{2}}$, 
Table 1 List of mathematical symbols
D: characteristic dimension of a rain measurement network

L: 1/e spatial decorrelation distance

$\mathrm{L}_{t}: 1 / e$ spatial decorrelation distance corresponding to $\mathrm{T} \times v$

$s$ : spatial lag along the horizontal

$S(\kappa)$ : spatial power spectrum

$S(\omega)$ : temporal power spectrum

$S_{t}(\kappa)$ : spatial power spectrum corresponding to the advection of the temporal power spectrum $S(\omega)$

$S_{s}(\omega)$ : temporal power spectrum correspond gin to the advection of the spatial power spectrum $S(\kappa)$

$S_{\text {total }}(\omega)$ : total power spectrum arising from the sum of the intrinsic temporal power spectrum and the advected spatial power spectrum

$S_{\text {total }}(\kappa)$ : total power spectrum arising from the sum of the intrinsic spatial power spectrum and the advected temporal power spectrum

$\mathrm{T}$ : 1/e decorrelation time

$\mathrm{T}_{s}: 1 / e$ decorrelation time corresponding to $L / v$

$v$ : advection speed

$t$ : temporal lag corresponding to $s / v$

$\rho(\tau)$ : intrinsic temporal autocorrelation function

$\rho(s)$ : intrinsic spatial autocorrelation function

$\rho_{t}(s)$ : spatial autocorrelation function corresponding to the advection of $\rho(\tau)$

$\rho_{s}(\tau)$ : temporal autocorrelation function corresponding to the advection of $\rho(s)$

$\rho_{\text {total }}(s)$ : total spatial autocorrelation function corresponding to $S_{\text {total }}(\kappa)$

$\rho_{\text {total }}(\tau)$ : total temporal autocorrelation function corresponding to $S_{\text {total }}(\omega)$

$\kappa$ : wave number

$\tau$ : time lag

$\omega$ : frequency

$\lambda$ : wavelength where $\omega$ is the frequency (here we use per second) and $\kappa$ is the wave number (here we use per meter).

When there is advection velocity $v$, the distance over which the 1/e temporal decorrelation occurs is $\mathbf{\Omega}_{t}=\mathfrak{I} \times v$ while $t=s / v$. Consequently, (1a) becomes

$\rho_{t}(s)=\exp \left(-\frac{s}{\mathbf{\Omega}_{t}}\right)$

so that

$S_{t}(\kappa)=\frac{2 \mathfrak{Q}_{t}^{2}}{\pi\left(1+\mathfrak{Q}_{t}^{2} \kappa^{2}\right)^{2}}$,

and, therefore, the total spatial spectrum becomes

$S_{\text {total }}(\kappa)=\frac{1}{2}\left[\frac{2 \mathfrak{Q}^{2}}{\pi\left(1+\mathfrak{Q}^{2} \kappa^{2}\right)^{2}}+\frac{2 \mathfrak{Q}_{t}^{2}}{\pi\left(1+\mathfrak{Q}_{t}^{2} \kappa^{2}\right)^{2}}\right]$.

On the other hand, when the advection of the spatial field (implicit in Fig. 1) is considered, then the time to decorrelation for that field will be $T_{s}=\mathrm{L} / v$ while $t=s / v$ so that $(1 \mathrm{~b})$ becomes $\rho_{s}(\tau)=\exp \left(-\frac{\tau}{\mathfrak{I}_{s}}\right)$

and

$S_{s}(\omega)=\frac{2 \mathfrak{I}_{s}^{2}}{\pi\left(1+\mathfrak{I}_{s}^{2} \omega^{2}\right)^{2}}$.

Therefore, the total temporal power spectrum becomes

$S_{\text {total }}(\omega)=\frac{1}{2}\left[\frac{2 \mathfrak{I}^{2}}{\pi\left(1+\mathfrak{I}^{2} \omega^{2}\right)^{2}}+\frac{2 \mathfrak{I}_{s}^{2}}{\pi\left(1+\mathfrak{I}_{s}^{2} \omega^{2}\right)^{2}}\right]$.

Obviously, by the Wiener-Khintchine, the correlation functions corresponding to (4) and (6) are then

$\rho_{\text {total }}(s)=\frac{1}{2}\left[\exp \left(-\frac{s}{\mathfrak{L}}\right)+\exp \left(-\frac{s}{\mathfrak{Q}_{t}}\right)\right]$

$\rho_{\text {total }}(\tau)=\frac{1}{2}\left[\exp \left(-\frac{\tau}{\mathfrak{I}}\right)+\exp \left(-\frac{\tau}{\mathfrak{I}_{s}}\right)\right]$ 
Clearly, advection alters what is being measured both temporally and spatially. Moreover, as an additional complication, temporal measurements are usually averaged over time in order to reduce fluctuations as discussed further in Jameson (2017). In its simplest and most common form, this is the convolution of box filter of sampling length $T$ having uniform weighting applied to a time series so that its Fourier transform is the well-known Sinc function which, in this case, turns out to be $\operatorname{Sinc}(\omega)=\sin (\omega T / 2) /(\omega T / 2)$ as illustrated in Fig. 2a for $T=600 \mathrm{~s}$. Because the Fourier transform of a convolution becomes the product of the Fourier transform of the two convolving functions, the temporal power spectrum in its most complete form for this model with advection is then given by:

$S_{\text {total }}(\omega)=\frac{1}{2}\left[\frac{2 \mathfrak{I}^{2}}{\pi\left(1+\mathfrak{I}^{2} \omega^{2}\right)^{2}}+\frac{2 \mathfrak{I}_{s}^{2}}{\pi\left(1+\mathfrak{T}_{s}^{2} \omega^{2}\right)^{2}}\right] \frac{\operatorname{Sinc}^{2}\left(\frac{T \omega}{2}\right)}{2 \pi}$,

where $T_{S}$ is a function of advection velocity, $v$ as presented above.

On the other hand, as described previously, the spatial measurements are subject to spectral filtering by the spatial dimension of the network (Jameson 2017) illustrated in Fig. 2b. For computational convenience this filter is well represented by the relation

$F_{s}=\left[1-\operatorname{Sinc}^{2}\left(4 \pi \mathfrak{D} \kappa^{2}\right)\right]$,

where $D$ is the dimension of the network. This filter is applied directly to the spatial power spectrum so that (4) becomes:

$$
\begin{aligned}
S_{\text {total }}(\kappa)= & \frac{1}{2}\left[\frac{2 \mathfrak{Q}^{2}}{\pi\left(1+\mathfrak{Q}^{2} \kappa^{2}\right)^{2}}+\frac{2 \mathfrak{Q}_{t}^{2}}{\pi\left(1+\mathfrak{Q}_{t}^{2} \kappa^{2}\right)^{2}}\right] \\
& \times\left[1-\operatorname{Sinc}^{2}\left(4 \pi \mathfrak{D} \kappa^{2}\right)\right] .
\end{aligned}
$$

This latter expression becomes even more complex when we also consider that temporal observations are often first averaged before calculating spatial characteristics so that the Sinc function must also be applied to the time observation before first converting to spatial $\kappa$. The temporal filtering Sinc function component is then $\operatorname{Sinc}^{2}\left(\frac{T \omega}{2}\right) \rightarrow \operatorname{Sinc}^{2}\left(\frac{T v K}{2}\right)$ so that (10) becomes:

$S_{\text {total }}(\kappa)=\left[\frac{2 \mathfrak{Q}^{2}}{\pi\left(1+\mathfrak{Q}^{2} \kappa^{2}\right)^{2}}+\frac{\mathfrak{Q}_{t}^{2} \operatorname{Sinc}^{2}\left(\frac{T \nu \kappa}{2}\right)}{\pi^{2}\left(1+\mathfrak{Q}_{t}^{2} \kappa^{2}\right)^{2}}\right] \times\left[1-\operatorname{Sinc}^{2}\left(4 \pi \mathfrak{D} \kappa^{2}\right)\right]$,

where $T$ and $v$ represent the sample interval mean advection velocity, respectively, along the line of instruments.
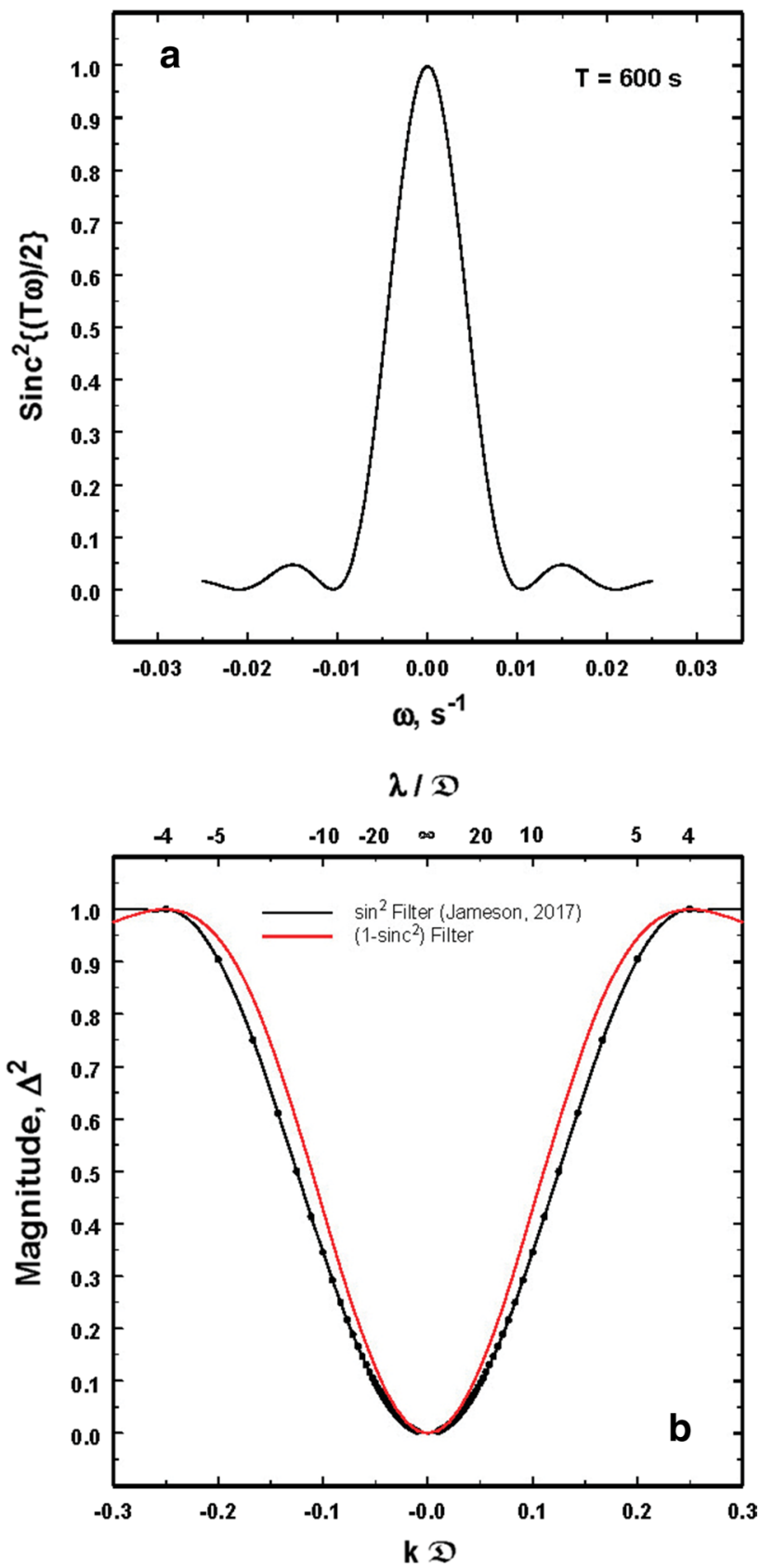

Fig. 2 a The Sinc function filter in time for a sample period of $T=600 \mathrm{~s}$ used in the analyses. It is a low pass filter. b The spatial filter defined in Jameson (2017) but now with the correct $x$-axis and now also fit by the $\left[1-\operatorname{Sinc}^{2}(4 \pi D \kappa)\right]$ where $D$ denotes the dimension of a network. It is a high-pass filter

Note that Mathematica ${ }^{\circledR}$ reveals that the filtered power spectra in (8), (10) and (11) all have deterministic inverse Fourier transforms which we use in subsequent analyses but which are much too large to be expressed here, although one can also numerically estimate the inverse Fourier transforms quite readily in Matlab ${ }^{\circledR}$, for example. 
In the next section we consider all of the power spectra given by (4), (6), (8), and (11) as well as their corresponding correlation functions as functions of advection velocities ranging from 0.1 to $9 \mathrm{~m} \mathrm{~s}^{-1}$. We begin by looking first at the effects of spatial advection on the temporal power spectra and correlation functions without any filtering as given by (6) and (7b). It is sufficient for the purpose here to present only examples having intrinsic $1 / e$ decorrelation time $T=600 \mathrm{~s}$ and decorrelation distance $L=2000 \mathrm{~m}$.

\section{Examples of the effects of advection and of sampling}

(a) Unfiltered temporal example using Eqs. (6) and (7b)

Figure $3 \mathrm{a}$ is a contour plot of the power (variance) spectra over the range of advection velocities. The most noteworthy features are the gradual upward slopes of the contours as the velocity increases as well as the bump of increased power at low frequencies and for velocities less than $1 \mathrm{~m} \mathrm{~s}^{-1}$. The reason for the second feature is that at low velocities, the movement of the spatial structures introduces longer $T_{s}$, i.e., introduces low frequencies, into (6). On the other hand, in contrast, as $v$ increases, $T_{s}$ decreases so that higher and higher frequencies play an increasingly important role.

These differences are also reflected in the power profiles presented in Fig. 3b. For the smallest advection velocity, the enhanced contributions at the lower frequencies are evident. In addition, the increasing importance of higher frequencies as the velocity increases is reflected in the change in shape as the contributions from lower frequencies decrease. Thus, the shapes of the power spectra depend upon the advection velocity.

This is also emphasized in Fig. 4 showing the fractional contribution by advection to the total spectral power. At low velocities, the contributions at lower frequencies are maximized, while those at higher frequencies are minimized. The opposite is true at larger advection velocities. Here it is worth noting that if the advection velocity were exactly $v=L / T$, then advection causes no deviation from the intrinsic power spectrum. In these examples, this occurs at a $v=3.33 \mathrm{~m} \mathrm{~s}^{-1}$.

Of course, changes in the power spectrum are also reflected as changes in the correlation functions as illustrated in Fig. 5. Since lower frequency contributions tend to enhance correlation while those at higher frequencies tend to decrease correlation, the tendency toward greater values in Fig. 5a at lower advection velocities at longer lags is understandable. Figure 5a also illustrates the decrease in correlation at larger $v$ as a downward slope of the contours as $v$ increases. These features are also clearly evident in the correlation profiles in Fig. 5b. As explained above, for
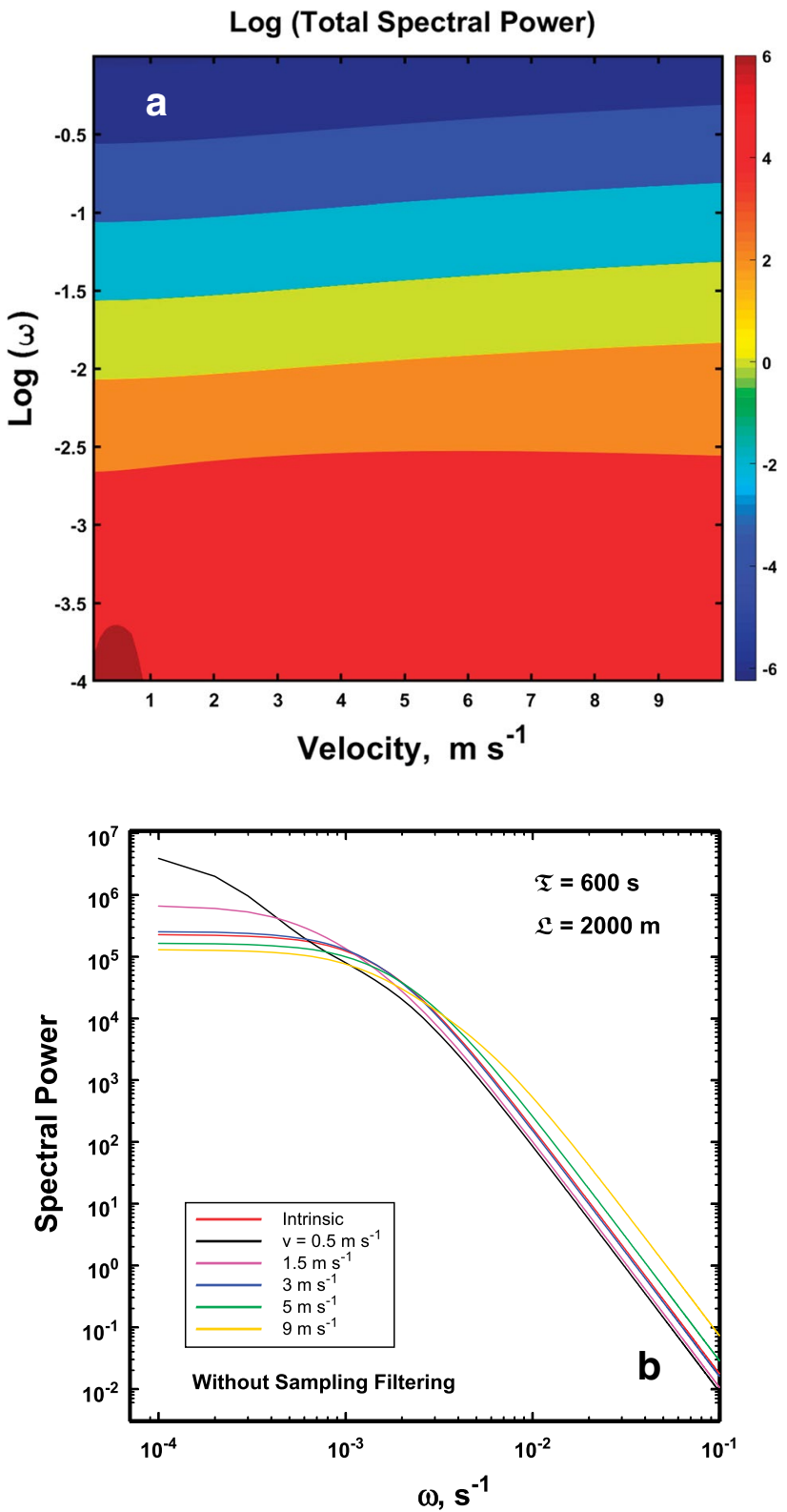

Fig. 3 a The logarithm (base 10) of the spectral powers (numbers on color bars) associated with temporal observations having an exponentially decreasing correlation function $\exp (-\tau / T)$ where $\tau$ is the time lag in combination with the advection at different velocities of spatial structures having an exponentially decreasing correlation function $\exp (-s / L)$, where $s$ is the separation distance. $T$ and $L$ select profiles are given in (b). Note that over $99.99 \%$ of the spectral power occurs for $\omega \leq 0.01 \mathrm{~s}^{-1}$

advection velocities less than about $3 \mathrm{~m} \mathrm{~s}^{-1}$, the times to decorrelation are significantly increased beyond the intrinsic value of $600 \mathrm{~s}$, so that at a $v=0.5 \mathrm{~m} \mathrm{~s}^{-1}$, the time to decorrelation is $1615 \mathrm{~s}$ or about 2.6 times the intrinsic value. On the other hand, for velocities greater than that, the opposite is true so that at $v=9 \mathrm{~m} \mathrm{~s}^{-1}$, the decorrelation time is reduced to 367 s or only 0.6 the intrinsic value. Thus, advection can 


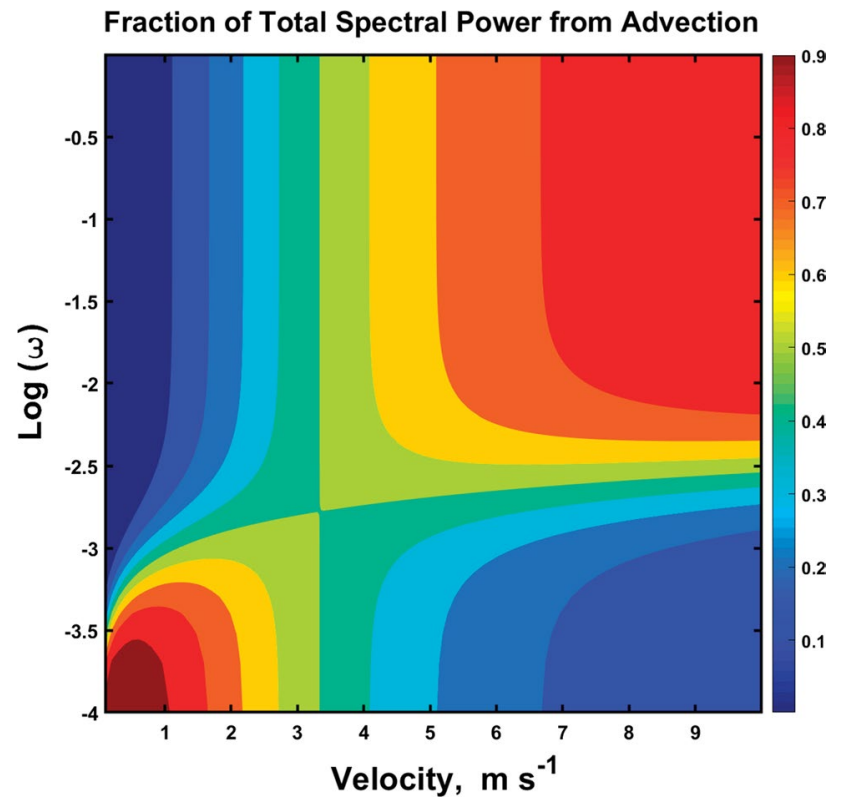

Fig. 4 Contours of the fraction (numbers on color bars) of the total temporal power spectra arising from the advection of the spatial structures presented in Fig. 3. Note that at low advection velocities, the advection of the spatial structures enhances low frequency contributions, while the opposite is true at larger advection velocities

significantly alter the intrinsic power spectra as well as the associated correlation functions of temporal observations.

\section{(b) Filtered temporal example using Eq. (8)}

When the temporal spectra are filtered, there are also significant changes to the power spectra and to the correlation functions for the reasons illustrated in Fig. 2a. The Sinc function acts to filter out higher frequencies so that the total power level decreases and a greater portion of the power is distributed into lower frequencies. This is illustrated for a sample time $T$ of $10 \mathrm{~min}$ in Fig. $6 \mathrm{a}$ where the reduction in the spectral powers at frequencies greater than around $0.02 \mathrm{~s}^{-1}$ is quite evident. Of course, this effect depends upon $T$ so that when $T$ is $1 \mathrm{~min}$, the effect is to reduce those frequencies around $0.1 \mathrm{~s}^{-1}$ and larger (Fig. 6b). Thus, the effects of filtering over $1 \mathrm{~min}$ are less than those over a 10 -min period. The results of applying Eq. (8) for 10-min sample filtering ( $T=600 \mathrm{~s}$ ) to the advected field in Fig. 3 are illustrated in Fig. 7.

Because of the filtering, oscillations appear and contributions from the higher frequencies show a sharper decline as shown in Fig. 7b than they did in Fig. 3b in which the latter closely followed the intrinsic profile. After filtering, they do not. Consequently, the relative importance of the lower frequencies increases, and this, in turn, impacts the correlation functions illustrated in Fig. 8. While the contours in Fig. 8a look similar to those in Fig. 5a, the profiles in Fig. 8b quite clearly illustrates the significant differences with all
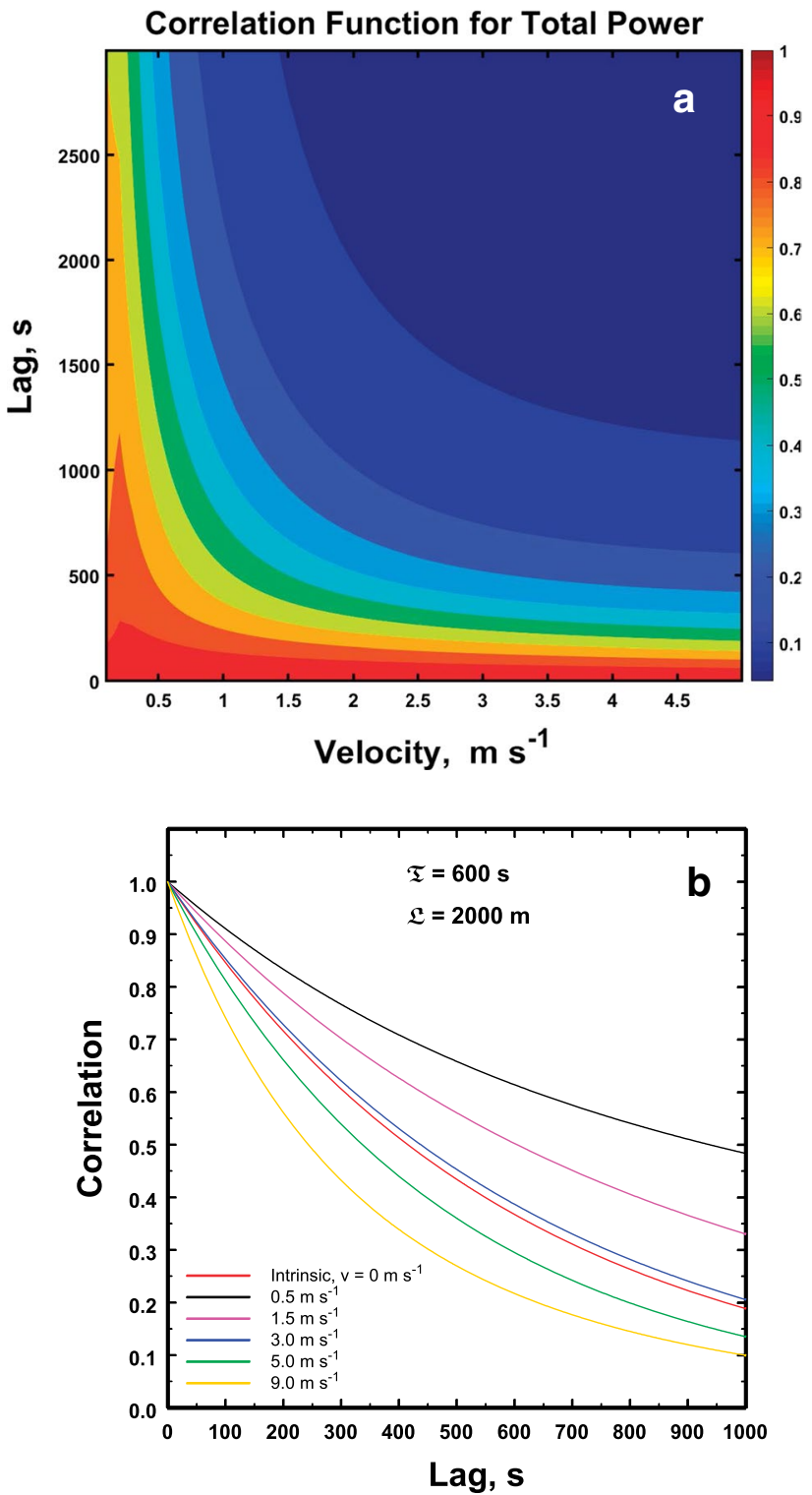

Fig. 5 a Contours of the correlation functions (numbers on color bars) associated with the power spectra in Fig. 3. As noted in Fig. 4, advection enhances lower frequencies and hence correlation at small velocities, while large velocities enhance larger frequencies and, hence, decorrelation as evident as well in the profiles in (b) at select velocities

correlation functions now lying above the intrinsic curve. Now the time to achieve 1/e decorrelation at $0.5 \mathrm{~m} \mathrm{~s}^{-1}$ and at $9 \mathrm{~m} \mathrm{~s}^{-1}$ are on the order of $7800 \mathrm{~s}$ and $1017 \mathrm{~s}$, respectively. Obviously, these values are much greater than the intrinsic value of $600 \mathrm{~s}$. When $T$ is only $60 \mathrm{~s}$, these values are reduced somewhat because of less averaging thereby leaving more of the higher frequencies so that, for example, the time to decorrelation for $v=9 \mathrm{~ms}^{-1}$ reduces to $1006 \mathrm{~s}$. Thus, in this example at least, whether $T$ is $60 \mathrm{~s}$ or $600 \mathrm{~s}$ is not terribly important. What is important is that the filtering 


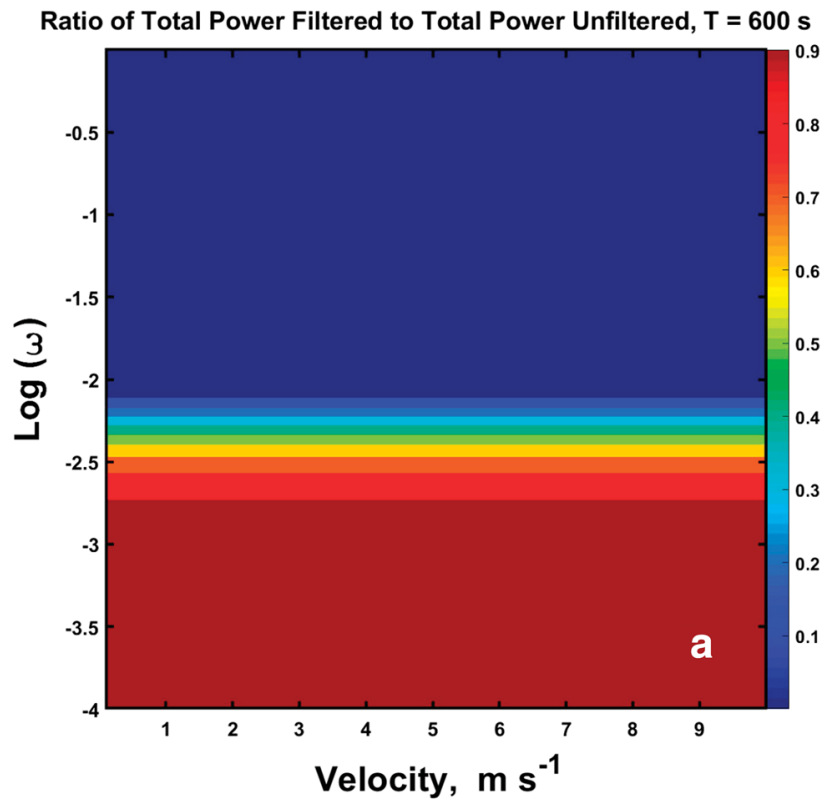

Ratio of Total Power Filtered to Total Power, $\mathbf{T}=60 \mathrm{~s}$

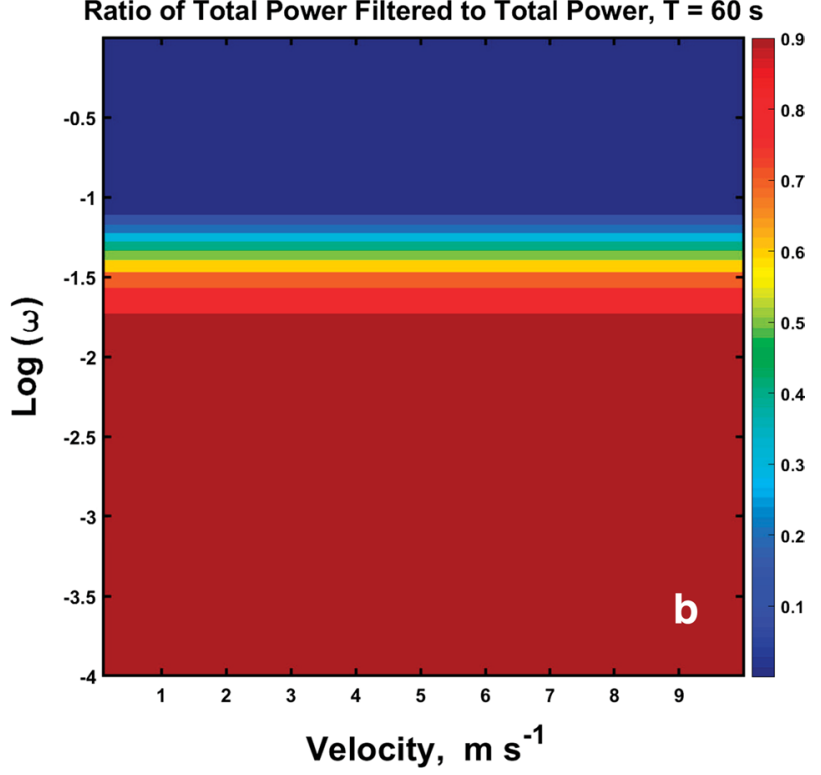

Fig. 6 The ratio of filtered power to the unfiltered power (numbers on color bars) using the Sinc function for two filter times, namely of $600 \mathrm{~s}$ (a) and for $T=60 \mathrm{~s} \mathrm{(b).} \mathrm{The} \mathrm{attenuated} \mathrm{frequencies} \mathrm{only}$ decrease from about $0.08 \mathrm{~s}^{-1}$ at $60 \mathrm{~s}$ down to $0.008 \mathrm{~s}^{-1}$ at $600 \mathrm{~s}$. Detailed calculations show that for the conditions set in this example, these differences produce very small changes between the two corresponding correlations. Consequently, in this rain model 1-min averaging has just as much impact as 10 min of averaging

of the higher frequencies by time averaging can significantly impact the correlation functions and power spectra.

(c) Unfiltered spatial example using Eqs. (4) and (7b)

When considering the effects of the advection of the temporal fluctuations over a network, the characteristic spatial
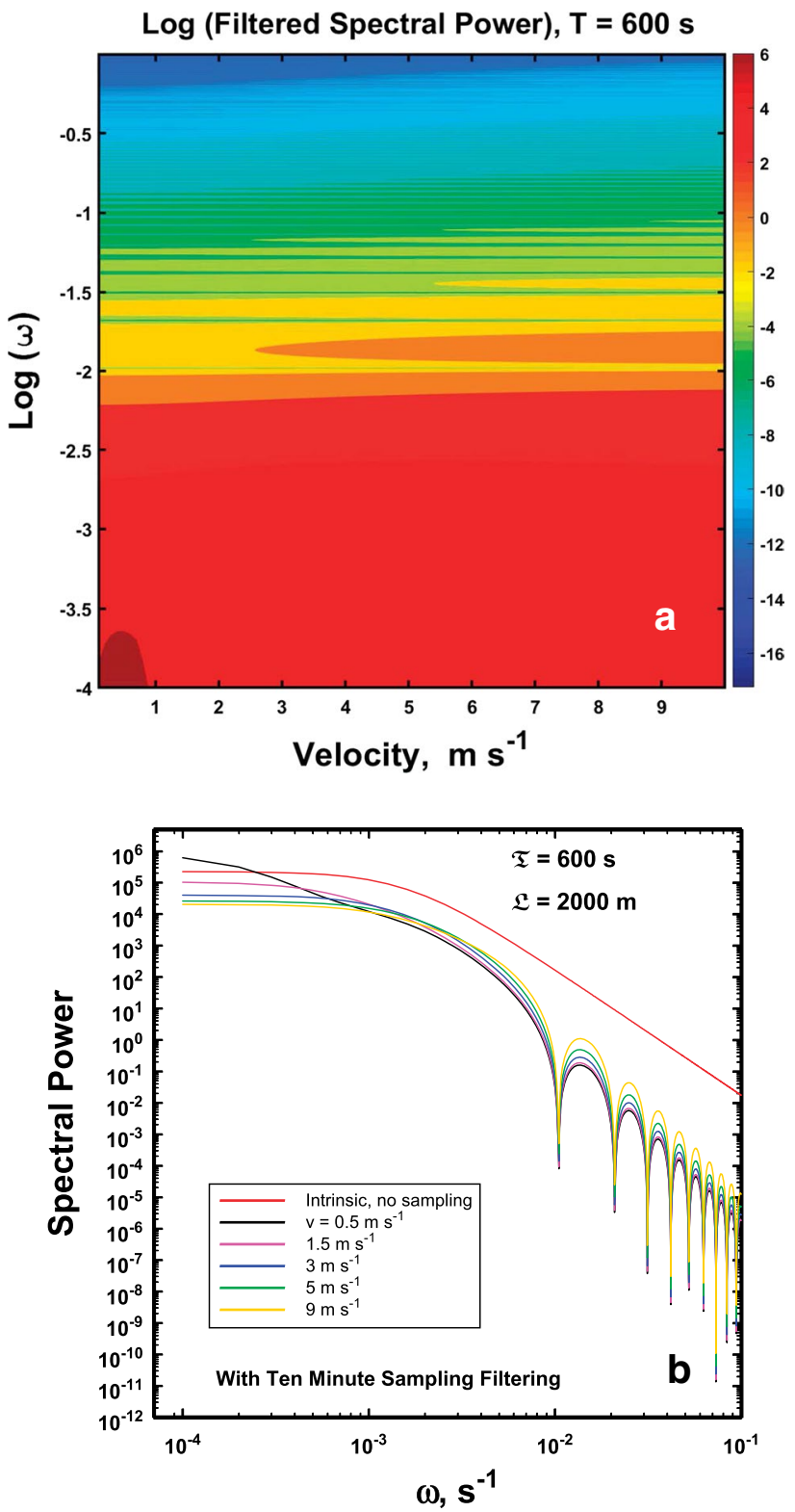

Fig. 7 a Logarithm (numbers on color bars) of the Sinc-filtered spectral power contours for the temporal exponential correlation function along with (b) select profiles. Note the reduced power levels and steeper profile changes as compared to those in Fig. $3 \mathrm{~b}$ as well as the oscillations introduced by the filtering. Note that over $99.99 \%$ of the spectral power occurs for $\omega \leq 0.01 \mathrm{~s}^{-1}$

scale becomes $L_{t}=v \times T$. Consequently, when $v$ is small, so is $L_{t}$. This means that there will be an increase in the importance of small-scale (large wave number) fluctuations. On the other hand, when $v$ is large, $L_{t}$ is also large so that the importance of larger scales (small wavenumbers) increases instead. The reverse is also true. These effects are illustrated in Fig. 9 where the increase in the power contribution at small velocities and large wave numbers is contrasted with the increase in power contributions at large velocities 

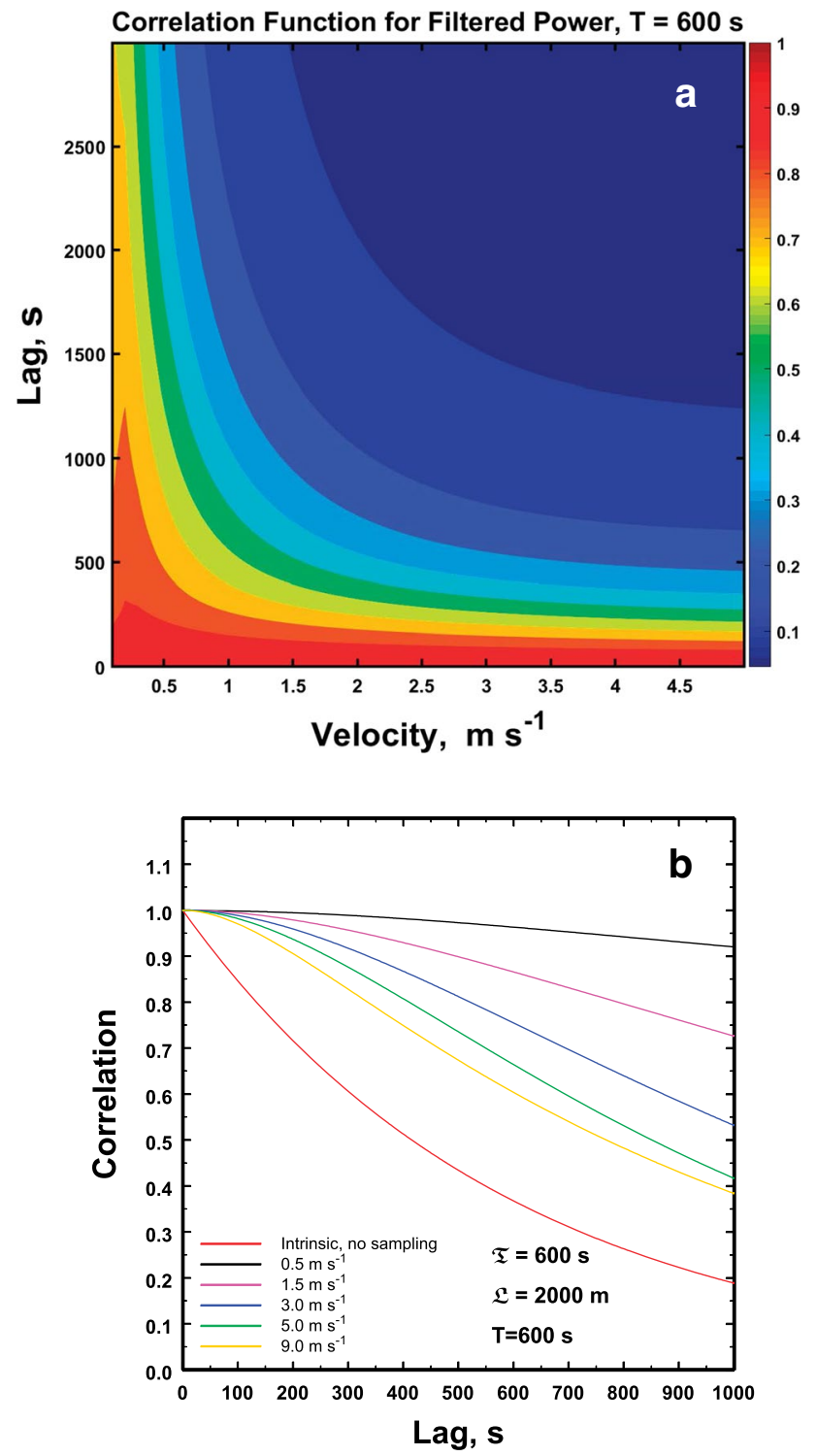

Fig. 8 Contours of the power-filtered correlation functions (numbers on color bars) in Fig. 7. Because the Sinc function is a low-pass filter, all the correlations are now enhanced beyond those in Fig. 5 and that of the intrinsic correlation function. Hence, observed correlation functions can be significantly affected by temporal averaging

and small wavenumbers. This alters the power spectra as indicated in Fig. 10a where the contours of power at large wavenumbers tilts upward at low velocities while the spectral powers increase at small wavenumbers (larger scales) at large velocities. Hence, the spectral power profiles change with a clockwise rotation with increasing velocities.

Obviously, these changes will also be reflected in the spatial correlation functions as illustrated in Fig. 11. At $v=0.5 \mathrm{~m} \mathrm{~s}^{-1}$, the separation to $1 / e$ decorrelation is now only 808 meters as compared to the intrinsic value of $2000 \mathrm{~m}$. In comparison, at $v=9 \mathrm{~m} \mathrm{~s}^{-1}$, that length has increased to $3295 \mathrm{~m}$ as shown in Fig. 11b. Thus, just as for the temporal

\section{Fraction of Total Spectral Power from Advection}

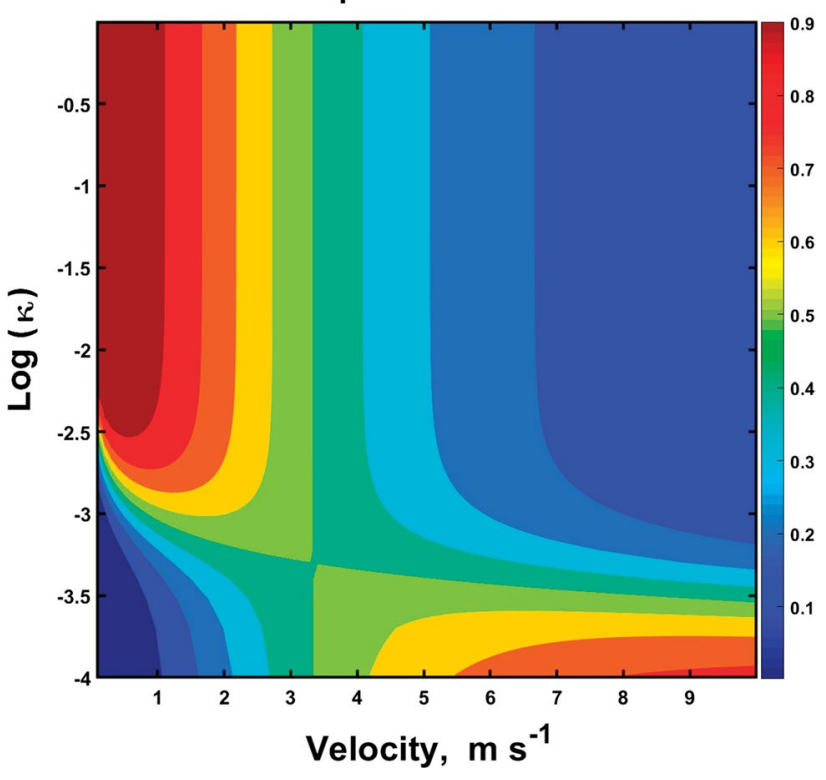

Fig. 9 Contours of the fraction (numbers on color bars) of the spectral power contributing to spatial observations from the advection of temporal fluctuations where $\kappa$ is the wave number. In contrast to Fig. 4, advection now contributes power to higher wavenumbers at low velocities tending to increase decorrelation with increasing lag more quickly, while at larger velocities, the contributions of more power at lower wave numbers tend to enhance correlation

observations, and even with this simple model it is clear that advection can potentially cause profound deviations from the intrinsic spectral power and correlation function. These changes are even more complicated when the effects of filtering are included as discussed next.

\section{(d) Filtered spatial example using Eq. (11)}

In spatial observations there are two sources of filtering. The most obvious is that of the finite dimension of the network as illustrated in Fig. $2 b$ in which the lower frequencies are strongly attenuated for wavelengths greater than about four times the network dimension (Jameson 2017). The less apparent filtering arises because of time averaging at the different locations in a network before being combined to estimate the spatial properties [e.g., see Ciach and Krajewski (2006)]. These two filters are given in (11).

The power spectra in Fig. 12 shows significant differences from the unfiltered example in Fig. 10 with a smaller contribution to larger wavenumbers at small velocities but a significant increase in the contributions to smaller wavenumbers at all velocities. This is quite apparent in Fig. 13. Such changes also significantly alter the associated correlation functions. These are reflected in the correlation functions are illustrated in Fig. 14a where the upward slopes of contours for velocities greater than 

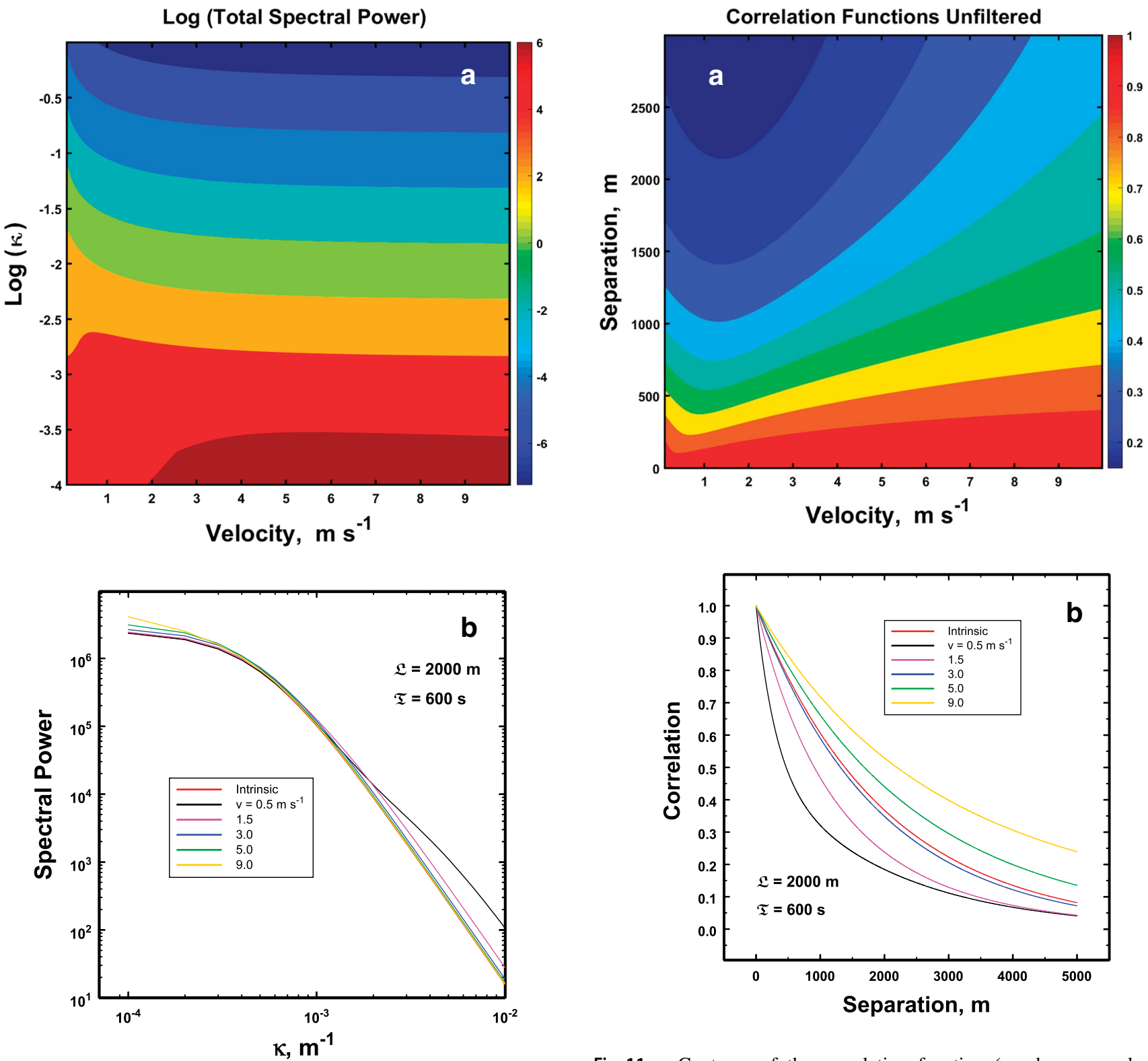

Fig. 10 a Contours of the logarithm of the spatial spectral power (numbers on color bars) combined with the advection of temporal fluctuations showing the enhanced powers at lower wave numbers and greater powers at larger $\kappa$ at smaller velocities. These changes are also reflected in the profiles in $\mathbf{b}$

$v=0.5 \mathrm{~m} \mathrm{~s}^{-1}$ are obvious. Furthermore, in Fig. $14 \mathrm{~b}$ even the unique velocity of $v=L / T$ of $3.3 \mathrm{~m} \mathrm{~s}^{-1}$ (approximately represented by the $3 \mathrm{~m} \mathrm{~s}^{-1}$ profile in the figure), now no longer traces the intrinsic correlation curve. Moreover, the separations to $1 / e$ decorrelation exceed $4 \mathrm{~km}$ at all velocities. All the correlation curves assume much more parabolic shapes reminiscent of the curves in Fig. 6 of Ciach and Krajewski (2006) and of that in Fig. 5 of Jaffrain and Berne (2012). Unfortunately, detailed comparisons are unwarranted because $\mathrm{T}$ is not given in either study and, of

course, the forms of the intrinsic correlation functions in those studies may well be different from the exponential functions assumed here.

\section{(e) Accounting for advection velocity}

As the above analyses indicate, it is important to try to account for precipitation advection. When available, observations of radar echo motion often provide a reasonable estimate of the advection vector $\mathbf{v}$. Perhaps one of the cleanest 
Total Filtered Spectral Power, $T=600 \mathrm{~s}, \mathfrak{D}=1000 \mathrm{~m}$
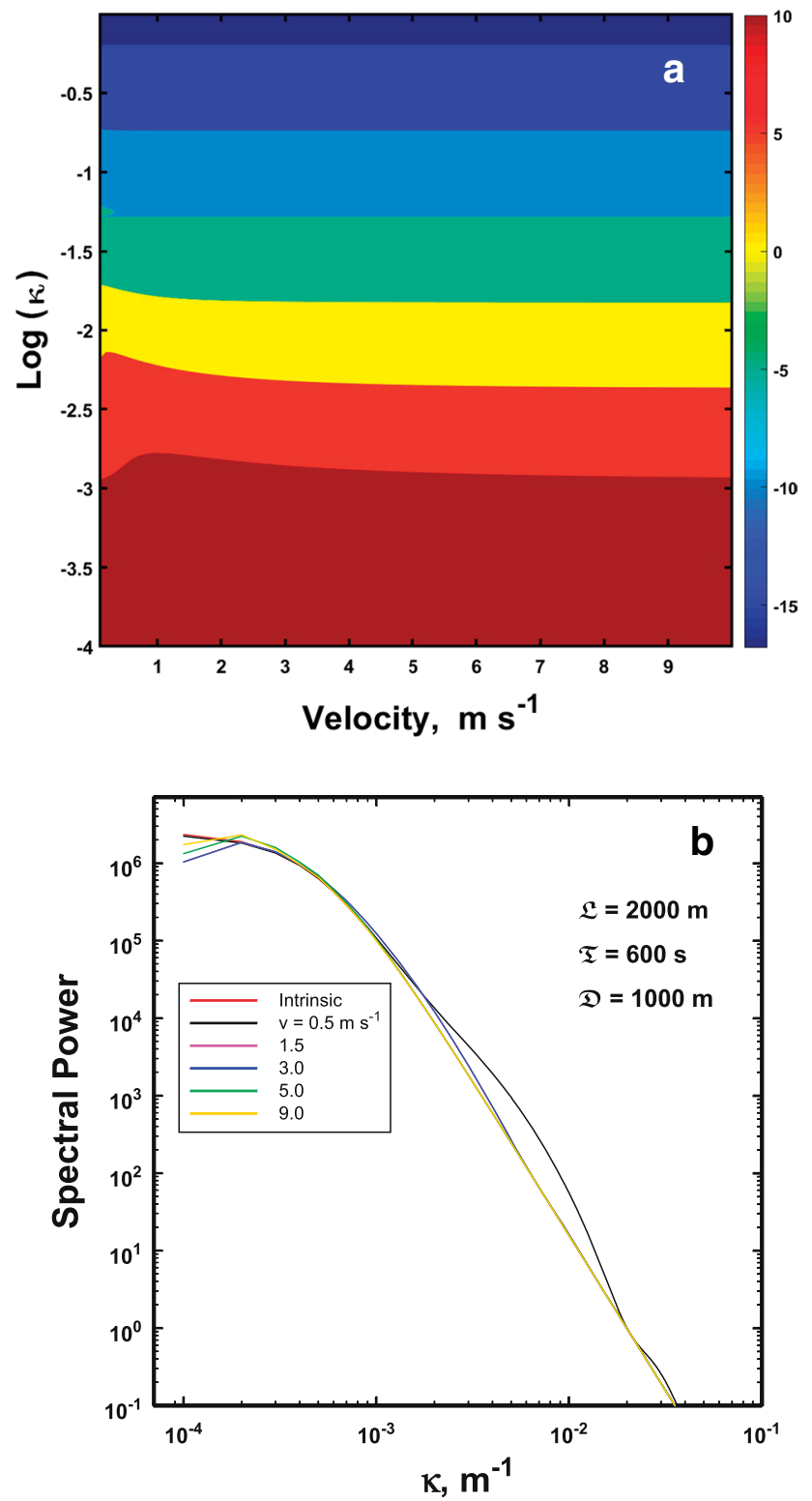

Fig. 12 a Contours of the logarithm of spatially and temporally filtered spatial power spectra (numbers on color bars) as discussed in the text. Compared to Fig. 10a, the enhanced powers at lower wavenumbers is obvious even at the very lowest advection velocities which still retain some contributions at higher wavenumbers as indicated in the profiles in $\mathbf{b}$

approaches to begin accounting for advection, then, is to use an estimate of $\mathbf{v}$, and then using a vehicle or drone moving at $\mathbf{v}$ to carry a detector for collecting temporal observations. In that way, $\mathbf{v}=0$, and the temporal correlation function and power spectra could then be estimated independently. Moreover, the form of this function could be used as a proxy for the form of the correlation function corresponding to the spatial measurements from a network. Thus, for example, in this particular model, after computing $L_{t}=|v| \times T$, one can

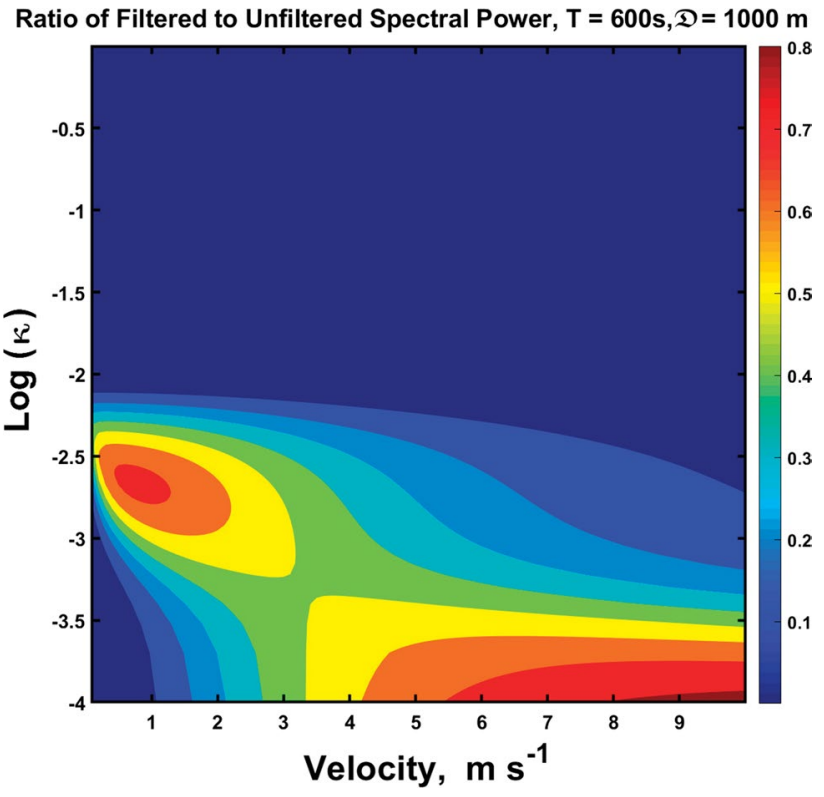

Fig. 13 Contours of the ratio of the filtered to unfiltered power spectra (numbers on color bars) as a function of advection velocity are illustrated. Compared to Fig. 9a, the enhanced contributions at lower wavenumbers at all velocities as well as the suppression of contributions for $\kappa<0.01 \mathrm{~m}^{-1}$ are obvious. This produces significant enhancements in the correlations as illustrated in the next figure

then subtract the component $\exp \left(-s / L_{t}\right)$ in $(7 \mathrm{a}, 7 \mathrm{~b})$ from the observed correlation function to derive the estimate of the actual spatial correlation function. Alternatively, one could also work with the power spectra after first dividing out the filters, which are known, and then using the Wiener-Khintchine theorem to derive the correlation functions. Trying to account for advection, however, is not a trivial matter and it will require additional thought and research.

\section{Concluding discussion}

Because of the multi-dimensional, multi- stochastic character of rain, one of the greatest challenges in the study of rain has always been that of measurements. Yet rain affects many aspects of human existence from the inconvenience of rained-out picnics to devastating floods. Consequently, such observations, even if challenging, remain of great interest.

This study shows just how difficult and misleading even the apparently straightforward observations of temporal and spatial correlation functions and power spectra might be even in the absence of statistical heterogeneity and/or nonstationarity. In particular, a simple mean velocity advection can produce a blending of the temporal and spatial statistical characteristics of the rain making it very difficult to extract the intrinsic, true spatial and temporal power spectra and correlational functions. 

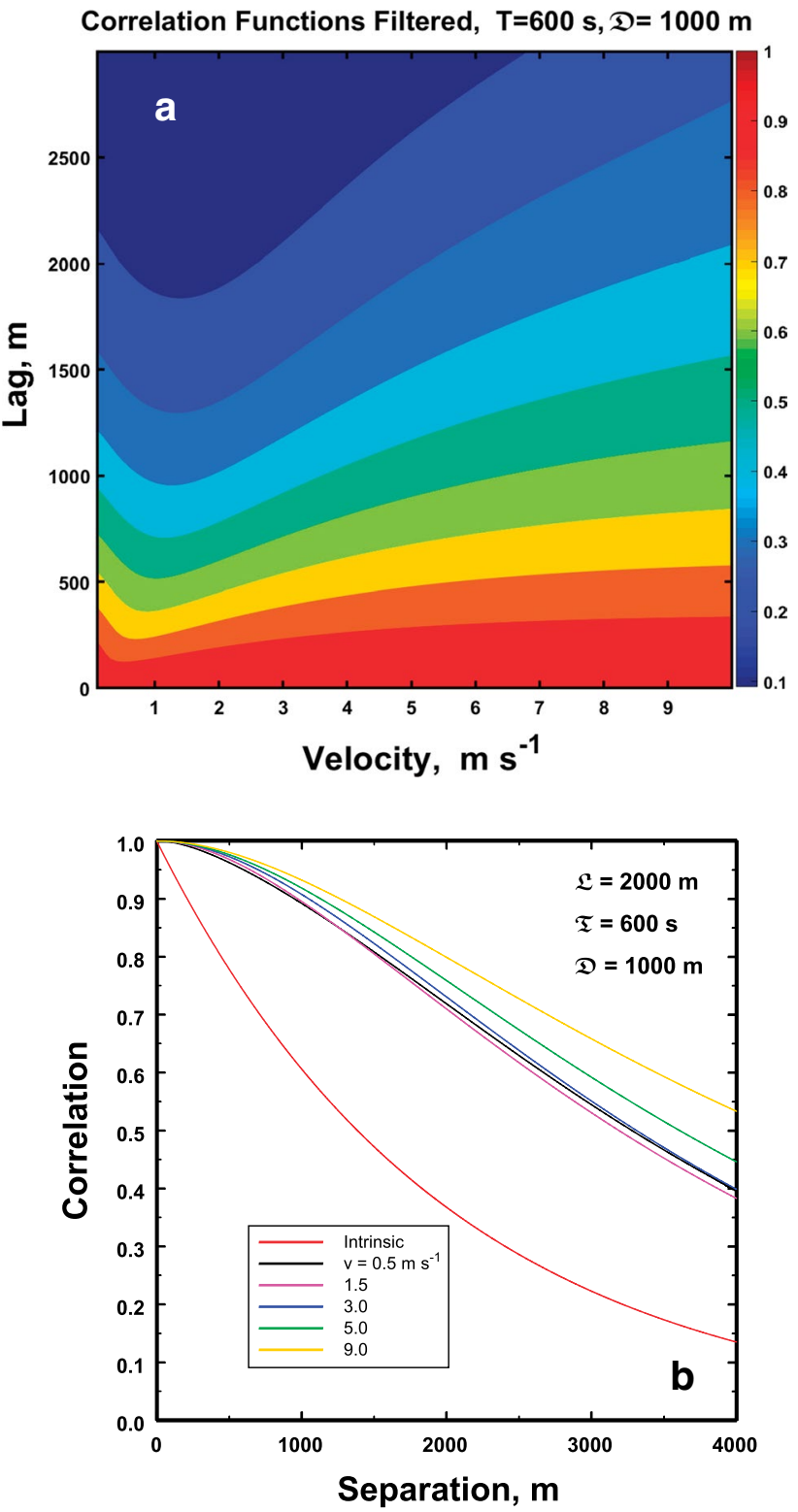

Fig. 14 a Contours of the correlation function (numbers on color bars) versus the advection velocity. Note the upward slope of the contours for all velocities greater than about $1 \mathrm{~m} \mathrm{~s}^{-1}$. At all velocities, however, the enhanced correlation compared to the intrinsic correlation function is evident in the profiles which are reminiscent, for example, of those found in Fig. 6 of Ciach and Krajewski (2006)

While this study focuses on a specific rain model, the following are likely valid regardless of the precise forms of the intrinsic correlation functions in time and space as long as they decrease with increasing lags. Specifically, the advection of the spatial variance structures during temporal observations at a location can produce either enhanced correlation and greater decorrelation times when the advection velocity is small, or it can lead to greater decorrelation and smaller decorrelation times when the advection velocity is substantial. The opposite appears to be true for the passage of temporal variance structures over a network, namely small advection velocities introduce higher spatial wavenumbers which lead to greater decorrelation, while larger velocities lead to enhance correlation because of increased contributions at lower wavenumbers. As illustrated in this work, these effects are also modulated by the filtering that occurs during the measurement process.

While it is important for a scientist to understand what is being measured, the original intent of this research was to find methods to circumvent the effects of advection on measurements of some of the basic statistical characteristics of rain, namely the spatial and temporal correlation functions and their associated power (variance) spectra often used to scale rain observations or numerical model outputs (e.g., Errico 1985; Berndtsson and Niemczynowicz 1988; Bell et al. 1990; Crane 1990; Harris et al. 2001; Germann and Zawadzki 2002; Parodi et al. 2011; de Lima et al. 2012; Ochoa-Rodriguez et al. 2015; Wong and Skamarock 2016). During this work, however, it became clear that achieving that objective requires additional measurements.

Consider (8). By using sufficiently short sample times $(T)$ the Sinc function approaches unity. Alternatively, it could also, in principle, be divided out of $S_{\text {total }}(\omega)$. The second term in the summation in (8) disappears as $v \rightarrow 0$ so that measurements must be made when $v$ is small. In all other cases one can attempt to estimate $v$ during the course of measurements, perhaps by using a radar or lidar to track echo motion over the location during observations.

With regard to (11) one can in principle remove the filtering effect of the grid size $D$ by dividing it out of $S_{\text {total }}(\kappa)$. In this case, however, the only way for removing the effect of the sample time is to make it small so that the Sinc function goes to unity. Finally, the second term goes to zero as $v \rightarrow$ 0 , so that one must again either make observations when $v$ is small or, preferably, estimate $v$ as suggested above. The practicality of achieving all of these possibilities remains to be explored.

Acknowledgements This work was supported by the National Science Foundation (NSF) under Grants AGS1823072 and AGS2001343.

Open Access This article is licensed under a Creative Commons Attribution 4.0 International License, which permits use, sharing, adaptation, distribution and reproduction in any medium or format, as long as you give appropriate credit to the original author(s) and the source, provide a link to the Creative Commons licence, and indicate if changes were made. The images or other third party material in this article are included in the article's Creative Commons licence, unless indicated otherwise in a credit line to the material. If material is not included in the article's Creative Commons licence and your intended use is not permitted by statutory regulation or exceeds the permitted use, you will need to obtain permission directly from the copyright holder. To view a copy of this licence, visit http://creativecommons.org/licenses/by/4.0/. 


\section{References}

Ahrens B, Beck A (2008) On upscaling of rain-gauge data for evaluating numerical weather forecasts. Meteorol Atmos Phys 99:155167. https://doi.org/10.1007/s00703-007-0261-8

Bell TL, Abdullah A, Martin RL, North GR (1990) Sampling errors for satellite-derived tropical rainfall: Monte Carlo study using a space-time stochastic model. J Geophys Res Atmos 95:21952205. https://doi.org/10.1029/JD095iD03p02195

Berndtsson R, Niemczynowicz J (1988) Spatial and temporal scales in rainfall analysis - some aspects and future perspectives. J Hydrol 100:293-313. https://doi.org/10.1016/0022-1694(88)90189-8

Ciach GJ, Krajewski WF (2006) Analysis and modeling of spatial correlation structure in small-scale rainfall in Central Oklahoma. Adv Water Resour 29:1450-1463. https://doi.org/10.1016/j.advwa tres.2005.11.003

Crane RK (1990) Space-time structure of rain rate fields. J Geophys Res Atmos 95:2011-2020. https://doi.org/10.1029/JD095iD03p 02011

de Lima MIP, Krajewski WF, de Lima JLMP (2012) Space-time precipitation from urban scale to global change. Adv Water Resour 45:1. https://doi.org/10.1016/j.advwatres.2012.06.001

Errico RM (1985) Spectra computed from a limited area grid. Mon Weather Rev 113:1554-1562. https://doi.org/10.1175/15200493(1985)113\%3c1554:SCFALA\%3e2.0.CO;2

Germann U, Zawadzki I (2002) Scale-dependence of the predictability of precipitation from continental radar images. Part I: description of the methodology. Mon Weather Rev 130:2859-2873. https://doi.org/10.1175/1520-0493(2002)130\%3c2859:SDOTP $\mathrm{O} \% 3 \mathrm{e} 2.0 . \mathrm{CO} ; 2$

Harris D, Foufoula-Georgiou E, Droegemeier KK, Levit JJ (2001) Multiscale statistical properties of a high-resolution precipitation forecast. J Hydrometeorol 2:406-418. https://doi.org/10.1175/15257541(2001)002\%3c0406:MSPOAH\%3e2.0.CO;2

Jaffrain J, Berne A (2012) Quantification of the small-scale spatial structure of the raindrop size distribution from a network of disdrometers. J Appl Meteorol Climatol 51:941-953. https://doi. org/10.1175/JAMC-D-11-0136.1

Jameson AR (2017) Spatial and temporal network sampling effects on the correlation and variance structures of rain observations. J Hydrometeorol 18:187-196

Jameson AR (2019) On the importance of statistical homogeneity to the scaling of rain. J Atmos Ocean Technol 36:1063-1078. https ://doi.org/10.1175/JTECH-D-18-0160.1

Jameson AR, Johnson DB (1990) Cloud microphysics and radar. In: Atlas D (ed) Radar in meteorology. American Meteorological Society, Boston, pp 323-340

Jameson AR, Larsen ML (2016) Estimates of the statistical twodimensional spatial structure in rain over a small network of disdrometers. Meteorol Atmos Phys. https://doi.org/10.1007/s0070 3-016-0438-0

Khintchine A (1934) Korrelationstheorie der stationaren stochastischen Prozesse. Math Ann 109:604-615. https://doi.org/10.1007/BF014 49156

Kostinski AB, Jameson AR (2000) On the spatial distribution of cloud particles. J Atmos Sci 57:901-915. https://doi.org/10.1175/15200469(2000)057\%3c0901:OTSDOC\%3e2.0.CO;2

Krajewski WF, Duffy CJ (1988) Estimation of correlation structure for a homogeneous isotropic random field: a simulation study. Comput Geosci 14:113-122. https://doi.org/10.1016/00983004(88)90055-6

Krajewski WF, Ciach GJ, Habib E (2003) An analysis of small-scale rainfall variability in different climatic regimes. Hydrol Sci J 48:151-162. https://doi.org/10.1623/hysj.48.2.151.44694
Larsen ML, Clark A, Noffke M et al (2010) Identifying the scaling properties of rainfall accumulation as measured by a rain gauge network. Atmos Res 96:149-158. https://doi.org/10.1016/j.atmos res.2009.12.008

Leblois E, Creutin J-D (2013) Space-time simulation of intermittent rainfall with prescribed advection field: adaptation of the turning band method: simulation of rainfall with advection field. Water Resour Res 49:3375-3387. https://doi.org/10.1002/wrcr.20190

Ochoa-Rodriguez S, Wang L-P, Gires A et al (2015) Impact of spatial and temporal resolution of rainfall inputs on urban hydrodynamic modelling outputs: a multi-catchment investigation. J Hydrol 531:389-407. https://doi.org/10.1016/j.jhydrol.2015.05.035

Parodi A, Foufoula-Georgiou E, Emanuel K (2011) Signature of microphysics on spatial rainfall statistics. J Geophys Res. https://doi. org/10.1029/2010JD015124

Raupach TH, Berne A (2016) Small-scale variability of the raindrop size distribution and its effect on areal rainfall retrieval. J Hydrometeorol. https://doi.org/10.1175/JHM-D-15-0214.1

Rebora N, Ferraris L, von Hardenberg J, Provenzale A (2006a) RainFARM: rainfall downscaling by a filtered autoregressive model. J Hydrometeorol 7:724-738. https://doi.org/10.1175/JHM517.1

Rebora N, Ferraris L, von Hardenberg J, Provenzale A (2006b) Rainfall downscaling and flood forecasting: a case study in the Mediterranean area. Nat Hazards Earth Syst Sci 6:611-619

Rosenfeld D, Wolff DB, Amitai E (1994) The window probability matching method for rainfall measurements with radar. J Appl Meteorol 33:682-693. https://doi.org/10.1175/15200450(1994)033\%3c0682:TWPMMF\%3e2.0.CO;2

Sauvageot H (1994) The probability density function of rain rate and the estimation of rainfall by area integrals. J Appl Meteorol 33:12551262. https://doi.org/10.1175/1520-0450(1994)033\%3c125 5:TPDFOR\%3e2.0.CO;2

Seed AW, Srikanthan R, Menabde M (1999) A space and time model for design storm rainfall. J Geophys Res Atmos 104:31623-31630. https://doi.org/10.1029/1999JD900767

Short DA, Wolff DB, Rosenfeld D, Atlas D (1993) A study of the threshold method utilizing raingage data. J Appl Meteorol 32:13791387. https://doi.org/10.1175/1520-0450(1993)032\%3c137 9:ASOTTM\%3e2.0.CO;2

Simpson J, Woodley WL (1975) Florida area cumulus experiments 1970-1973 rainfall results. J Appl Meteorol 14:734-744. https ://doi.org/10.1175/1520-0450(1975)014\%3c0734:FACER $\mathrm{R} \% 3 \mathrm{e} 2.0 . \mathrm{CO} ; 2$

Steiner M, Smith JA (2004) Scale dependence of radar-rainfall rates-an assessment based on raindrop spectra. J Hydrometeorol 5:1171-1180

Tokay A, Bashor PG (2010) An experimental study of small-scale variability of raindrop size distribution. J Appl Meteorol Climatol 49:2348-2365. https://doi.org/10.1175/2010JAMC2269.1

Wiener N (1930) Generalized harmonic analysis. Acta Math 55:117258. https://doi.org/10.1007/BF02546511

Wong M, Skamarock WC (2016) Spectral characteristics of convectivescale precipitation observations and forecasts. Mon Weather Rev 144:4183-4196. https://doi.org/10.1175/MWR-D-16-0183.1

Zawadzki II (1973) Statistical properties of precipitation patterns. J Appl Meteorol 12:459-472

Publisher's Note Springer Nature remains neutral with regard to jurisdictional claims in published maps and institutional affiliations. 